\section{Michigan Technological \begin{tabular}{ll}
\hline $\mathbf{1} 8 \mathbf{8} 5$ & University \\
\hline
\end{tabular}}

Michigan Technological University Digital Commons @ Michigan Tech

8-31-2015

\title{
Controlling optical absorption in metamaterial absorbers for plasmonic solar cells
}

\author{
Wyatt Adams \\ Michigan Technological University \\ Ankit Vora \\ Michigan Technological University \\ Jephias Gwamuri \\ Michigan Technological University \\ Joshua M. Pearce \\ Michigan Technological University \\ Durdo O. Guney \\ Michigan Technological University
}

Follow this and additional works at: https://digitalcommons.mtu.edu/materials_fp

Part of the Materials Science and Engineering Commons

\section{Recommended Citation}

Adams, W., Vora, A., Gwamuri, J., Pearce, J. M., \& Guney, D. O. (2015). Controlling optical absorption in metamaterial absorbers for plasmonic solar cells. SPIE Proceedings, 9546. http://dx.doi.org/10.1117/ 12.2190396

Retrieved from: https://digitalcommons.mtu.edu/materials_fp/105

Follow this and additional works at: https://digitalcommons.mtu.edu/materials_fp

Part of the Materials Science and Engineering Commons 


\title{
Controlling Optical Absorption in Metamaterial Absorbers for Plasmonic Solar Cells
}

\author{
Wyatt Adams $^{\mathrm{a}}$, Ankit Vora ${ }^{\mathrm{a}}$, Jephias Gwamuri ${ }^{\mathrm{b}}$, Joshua M. Pearce ${ }^{\mathrm{a}, \mathrm{b}}$, and Durdu Ö. Güney*a \\ a Department of Electrical and Computer Engineering, Michigan Technological University, \\ Houghton, MI 49931, USA; ${ }^{\mathrm{b} D e p a r t m e n t}$ of Materials Science and Engineering, Michigan \\ Technological University, Houghton, MI 49931, USA.
}

\begin{abstract}
Metals in the plasmonic metamaterial absorbers for photovoltaics constitute undesired resistive heating. However, tailoring the geometric skin depth of metals can minimize resistive losses while maximizing the optical absorbance in the active semiconductors of the photovoltaic device. Considering experimental permittivity data for $\operatorname{In}_{\mathrm{x}} \mathrm{Ga}_{1-\mathrm{x}} \mathrm{N}$, absorbance in the semiconductor layers of the photovoltaic device can reach above $90 \%$. The results here also provides guidance to compare the performance of different semiconductor materials. This skin depth engineering approach can also be applied to other optoelectronic devices, where optimizing the device performance demands minimizing resistive losses and power consumption, such as photodetectors, laser diodes, and light emitting diodes.
\end{abstract}

Keywords: Metamaterials, Plasmonics, Perfect Absorbers, Solar Energy, Photovoltaics

\section{INTRODUCTION}

Optical enhancement of solar photovoltaic (PV) devices using conventional materials has previously been employed as a method of increasing device efficiency, though advances in this area have begun to slow. Metamaterials have shown promise as a way to engineer the optical properties of materials by manipulating the geometric parameters of subwavelength features ${ }^{1}$. The advent of metamaterials has provided unprecedented flexibility in manipulating light and producing new functionalities such as diffraction-unlimited imaging ${ }^{2}$ and lithography ${ }^{3}$, illusion optics ${ }^{4}$, optical analogue simulators $^{5,6}$, very low profile antennas ${ }^{7,8}$, solar photovoltaics ${ }^{9,10}$, metaspacers ${ }^{11}$, and more recently quantum information processing ${ }^{12,13}$. Particularly in PV, metamaterial technology offers a new degree of freedom for scientists and engineers to pursue enhancement of solar cells ${ }^{14}$.

Another approach to optical enhancement called plasmonics has also been considered, which enhances light absorption by exciting surface plasmons, quasiparticles of electron density fluctuations confined to a conducting surface, resulting in light-coupled surface plasmon polaritons $(\mathrm{SPP})^{25}$. The excitation of these SPPs result in enhanced electromagnetic fields which can improve the optical performance in devices such as PV devices, light-emitting diodes, perfect absorbers, waveguides, and others ${ }^{16-20}$. This approach to absorption enhancement shows promise for extremely thin film devices as

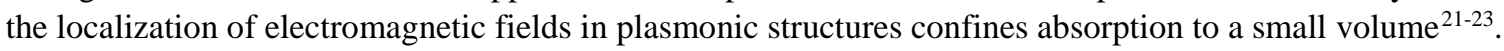

It is possible to combine both metamaterial and plasmonic approaches to engineer plasmonic perfect meta-absorbers tailored to a specific region of the electromagnetic spectrum which exhibit "perfect" absorbance and greatly enhance optical absorption over the region of interest. These absorbers can exhibit a number of optical properties, such as broadband absorption $^{24-29}$, wide angle-of-incidence absorption ${ }^{24,25,27,29-31}$, and polarization-independent absorption ${ }^{27-30,32}$. Despite this promise, perfect absorbers have inherent Ohmic loss (i.e., heating) ${ }^{9}, 33,34$, which decreases the efficiency of the electrical conversion.

Previously, the bulk skin depth effect has been used to exchange Ohmic losses in metamaterial absorbers for useful dielectric absorption in PV devices ${ }^{9}$. This method is effective in trapping incident electromagnetic waves in a semiconductor used in PV devices. Based on the observation of this phenomenon, a systematic approach to applying plasmonic perfect meta-absorbers and the bulk skin depth effect to real semiconductor materials is desired so that device

Active Photonic Materials VII, edited by Ganapathi S. Subramania, Stavroula Foteinopoulou, Proc. of SPIE Vol. 9546, 95461M - @ 2015 SPIE · CCC code: 0277-786X/15/\$18 · doi: 10.1117/12.2190396 
geometries can be engineered and optimized for the electromagnetic properties of the semiconductor active layer in photovoltaic devices. Here we present the results of optimizing the geometric and dielectric parameters of plasmonic perfect meta-absorbers for application to $\mathrm{PV}$ using indium gallium nitride $\left(\mathrm{In}_{\mathrm{x}} \mathrm{Ga}_{1-\mathrm{x}} \mathrm{N}\right)$ as an example.

\section{METHODS}

The study was carried out by performing electromagnetic simulations of plasmonic perfect meta-absorber structures in the finite element method (FEM) software COMSOL Multiphysics Version 4.4. The general two-dimensional absorber structure used can be seen in Fig. 1.

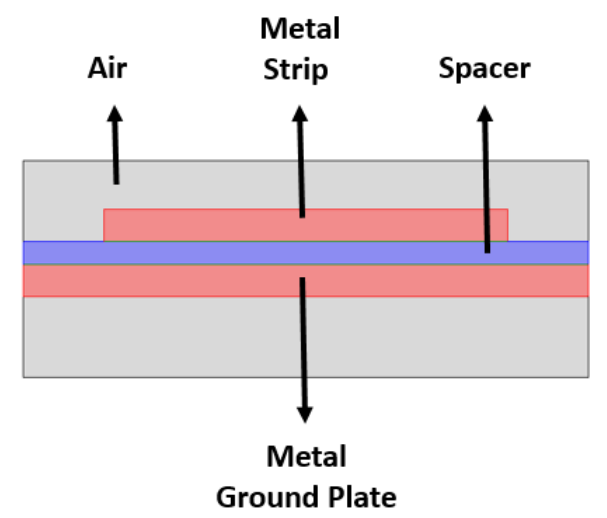

Figure 1. COMSOL two-dimensional plasmonic perfect meta-absorber model. A port at the top boundary of the model produces an x-polarized (i.e., parallel to the interfaces) electromagnetic wave normally incident on the absorber structure, which consists of a metallic strip, dielectric spacer, and metallic ground plate from top to bottom embedded in free space. The Drude model for gold was used for the metallic components. The areas separating the absorber from the top and bottom ports are modeled as air, and periodic boundary conditions are implemented on the left and right sides of the model. For this study, the thickness and width of the metallic strip in addition to the dielectric permittivity were modified to optimize the absorbance and tune the resonant frequency.

$\mathrm{In}_{\mathrm{x}} \mathrm{Ga}_{1-\mathrm{x}} \mathrm{N}$, which really has come under renewed interest as a candidate material of high-efficiency multi-junction PV devices due to bandgap engineering between $0.7 \mathrm{eV}$ and $3.4 \mathrm{eV}$ by varying the $\mathrm{x}^{35-39}$ is used as an example. In this case a single bandgap material of $\operatorname{In}_{0.54} \mathrm{Ga}_{0.46} \mathrm{~N}$ is analyzed. The structure consists of a metallic strip, spacer layer, and ground plate. The metallic strip and ground plate were modeled using the Drude model for gold, with a plasma frequency of 2100 $\mathrm{THz}$ and a collision frequency of $19 \mathrm{THz}$. Periodic boundary conditions are implemented at the left and right boundaries of the model. The dielectric spacer was modeled as a lossy material with a complex relative permittivity $\hat{\varepsilon}(\omega)$ given by Eq. 1:

$$
\hat{\varepsilon}(\omega)=\varepsilon^{\prime}(\omega)-j \varepsilon^{\prime \prime}(\omega)
$$

Table 1 . Real permittivity $\varepsilon^{\prime}$, frequency f, and reference geometric parameters of the simulated plasmonic perfect metaabsorbers displaying perfect absorbance in the neighborhood of $f$, where ts is the strip thickness, tg is the ground plate thickness, $\mathrm{d}$ is the dielectric thickness, $\mathrm{w}$ is the strip width, and $\mathrm{L}$ is the unit cell period.

\begin{tabular}{|l|c|l|l|l|l|l|l|}
\hline & $\varepsilon^{\prime}$ & $\mathrm{f}(\mathrm{THz})$ & $\operatorname{ts}(\mathrm{nm})$ & $\operatorname{tg}(\mathrm{nm})$ & $\mathrm{d}(\mathrm{nm})$ & $\mathrm{w}(\mathrm{nm})$ & $\mathrm{L}(\mathrm{nm})$ \\
\hline Point 1 & 0.86 & 145 & 15 & 60 & 14 & 315 & 400 \\
\hline Point 2 & 4.59 & 387 & 10 & 60 & 12 & 55 & 150 \\
\hline Point 3 & 0.86 & 387 & 5 & 60 & 12 & 79 & 150 \\
\hline Point 4 & 4.59 & 145 & 33 & 60 & 14 & 203 & 400 \\
\hline
\end{tabular}


Four permittivity and frequency points were considered corresponding to the $\varepsilon^{\prime}$ of $\operatorname{In}_{0.54} \mathrm{Ga}_{0.46} \mathrm{~N}$ at $145 \mathrm{THz}$ of 0.86 and at $387 \mathrm{THz}$ of 4.59 as given in Ref. [9]. The other two points chosen were an $\varepsilon^{\prime}$ of 0.86 at $387 \mathrm{THz}$ and 4.59 at $145 \mathrm{THz}$. For each point, a reference geometry with $\varepsilon^{\prime \prime}=0$ was selected as the starting structure to be optimized. These geometries were selected to exhibit the desired resonant frequency and $\varepsilon^{\prime}$ value. The four chosen points and the reference geometric parameters for the absorbers are given in Table 1.

An example absorbance spectrum for the reference structure corresponding to point 1 can be seen in Fig. 2(a). The metallic structure exhibits perfect absorbance at around $180 \mathrm{THz}$, with most of the absorption occurring in the metallic strip.

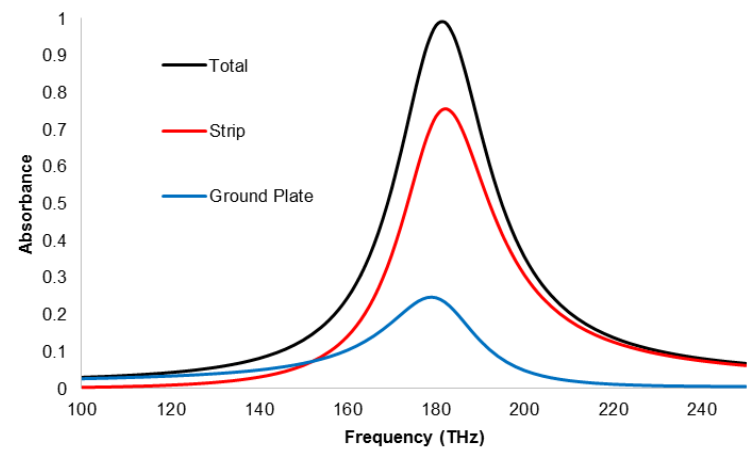

(a)

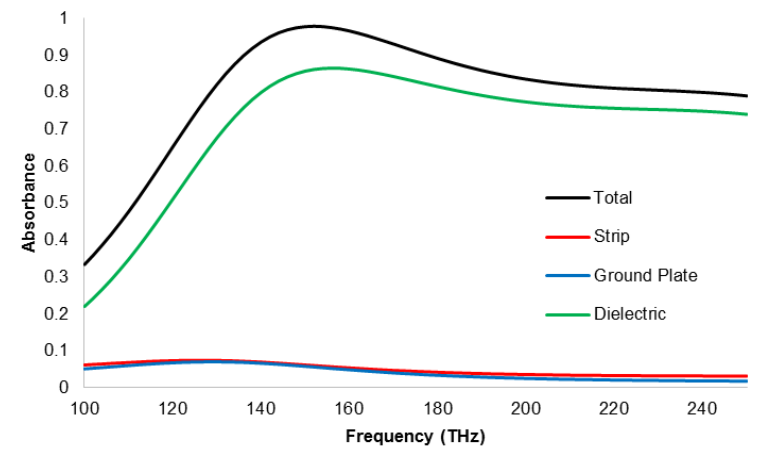

(b)

Figure 2. Point 1 absorbance for the reference structure (a) and the optimized structure with dielectric absorbance (b). In (b) it can be seen that an absorbance $>0.85$ can be recovered in the lossy dielectric layer.

To optimize the absorbance in the dielectric layer, the bulk skin depth effect ${ }^{9}$ was employed while simultaneously introducing a nonzero loss term $\varepsilon^{\prime \prime}$ into the dielectric permittivity. To do this, the thickness of the metallic strip was increased in 5-10 nm steps, and the $\varepsilon^{\prime \prime}$ value which corresponded to the maximum absorbance observed for each strip thickness was recorded. From this information, a plot of optimum $\varepsilon^{\prime \prime}$ versus strip thickness can be constructed. This plot for points 1-4 along with the corresponding dielectric absorbance will be discussed in detail below in the Results section. An example optimized spectrum for point 1 is shown in Fig. 2(b).

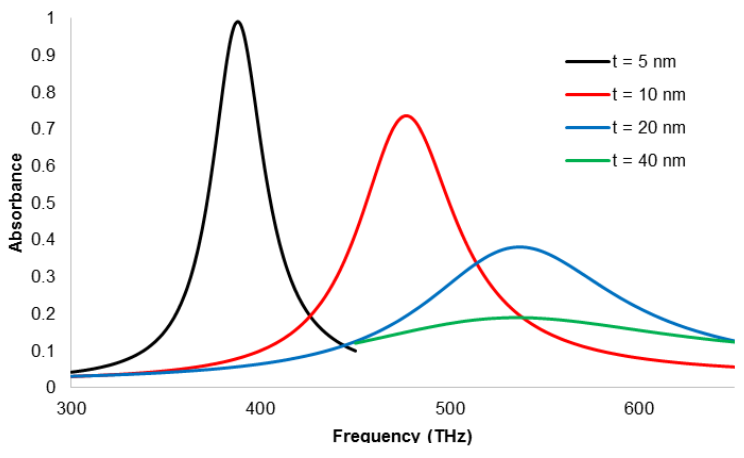

(a)

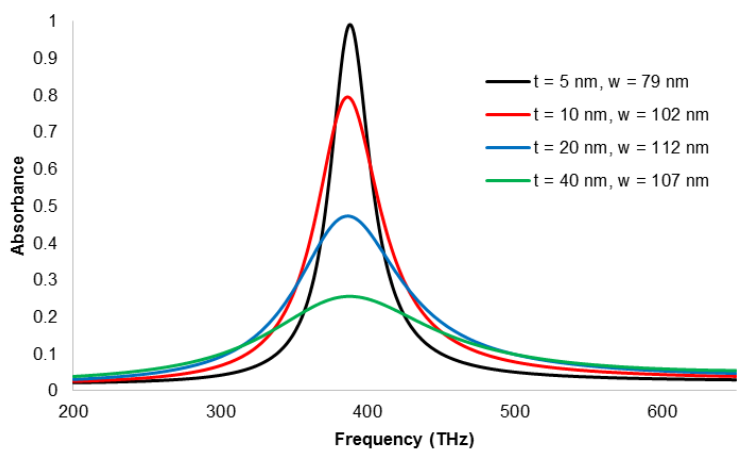

(b)

Figure 3. Absorbance spectra for point 3 at strip thicknesses t ranging from 5-40 nm. In (a), a large shift in the resonant frequency was observed as the strip thickness was varied. This shift was compensated by adjusting the strip width w using Eq. 2 , and the results of the tuning are shown in (b). It was observed that the resonant frequency without implementing the tuning procedure began to return closer to $387 \mathrm{THz}$ for higher strip thicknesses, which explains the lower $\mathrm{w}$ value for $\mathrm{t}=40 \mathrm{~nm}$ compared to $\mathrm{t}=20 \mathrm{~nm}$. 
For point 3, tuning of the structure was required due to a large shift in the resonant frequency as the strip thickness was increased. To do this, the width of the metallic strip was adjusted to compensate for the frequency shift following approximately an LC circuit model with the resonant frequency $\omega_{0}$ of the absorber given by:

$$
\omega_{0}=\frac{1}{\sqrt{L C}}
$$

where $\mathrm{L}$ is the effective inductance, and $\mathrm{C}$ is the effective capacitance. The "capacitor" in the absorber can be thought of as a parallel plate capacitor comprised of the metallic strip and the ground plate. Therefore, increasing the width of the strip would lead to an increase in the effective capacitance, thereby decreasing the resonant frequency. The absorbance spectra for point 3 are shown in Fig. 3 in order to illustrate the frequency tuning procedure. In Fig. 3, the LC model approach is used to shift the resonant frequency of the absorber at higher strip thicknesses back to $387 \mathrm{THz}$. This approach allowed for optimization of the dielectric absorbance for point 3 .

Table 2. Optimized parameters of the plasmonic perfect meta-absorbers operating at the points in Table 1, where Amax is the maximum absorbance in the lossy dielectric layer. Point 3 displays the highest maximum absorbance while also requiring the largest $\varepsilon^{\prime \prime}$. These results are further illustrated in Figs. 4 and 5.

\begin{tabular}{|l|c|c|c|c|}
\hline & $\varepsilon^{\prime \prime}$ & ts $(\mathrm{nm})$ & $\mathrm{w}(\mathrm{nm})$ & Amax \\
\hline Point 1 & 1.075 & 205 & 315 & 0.869 \\
\hline Point 2 & 0.95 & 110 & 55 & 0.808 \\
\hline Point 3 & 2.00 & 70 & 110 & 0.936 \\
\hline Point 4 & 0.75 & 115 & 203 & 0.622 \\
\hline
\end{tabular}
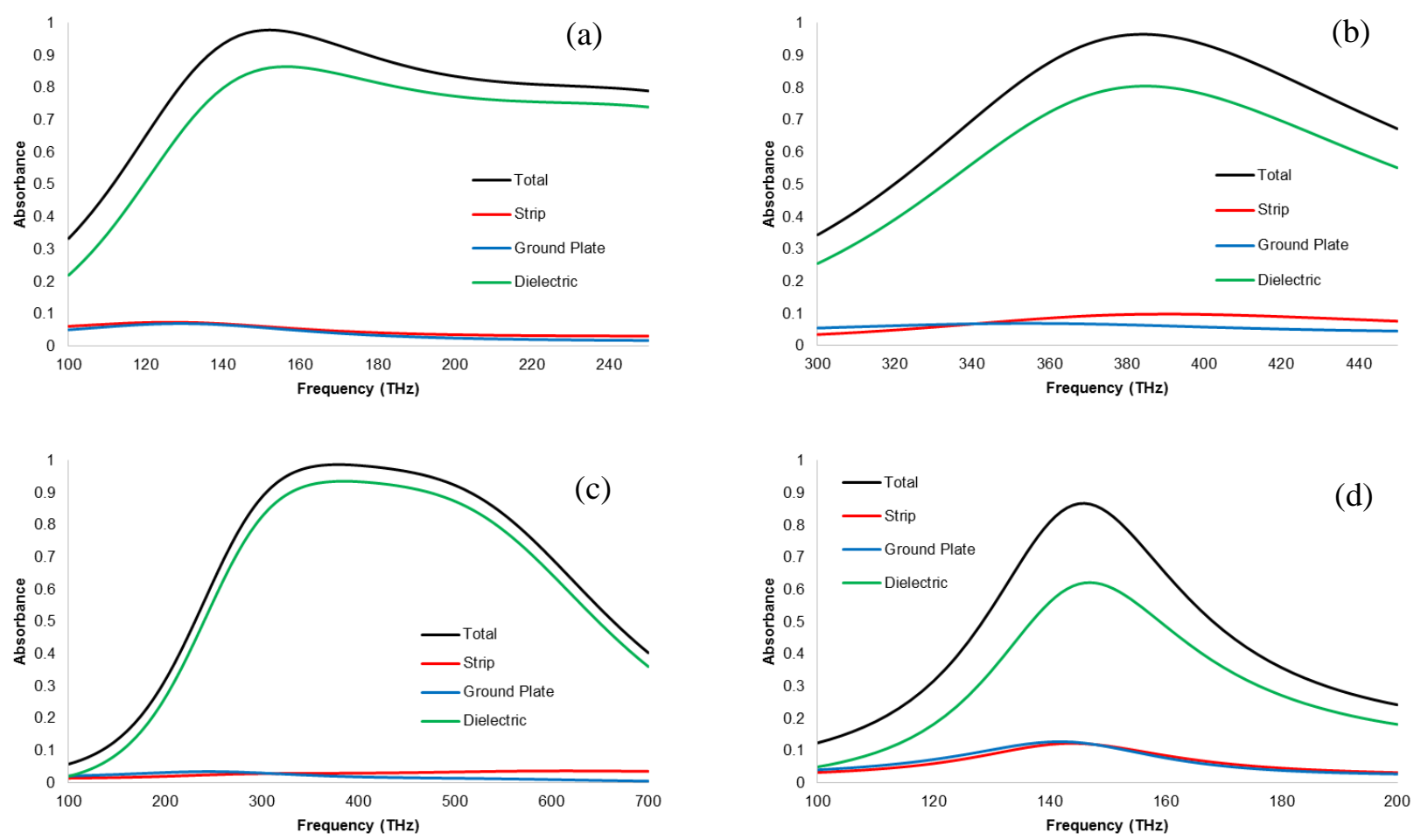

Figure 4. Absorbance spectra optimized for dielectric layer absorption corresponding to the four absorber points 1-4 (a-d) described in Table 1. Comparing to Fig. 2(a), it can be seen that the metallic absorption has been transferred from the metallic strip and ground plate to the dielectric layer by introducing a nonzero $\varepsilon^{\prime \prime}$ term and increasing the strip thickness to make use of the bulk skin depth effect. From the figure it can be seen that each optimization results in a different maximum absorbance as well as bandwidth, with Point 3 displaying the highest maximum absorbance. 


\section{RESULTS}

The optimized structure parameters are given in Table 2, obtained using the optimization procedure outlined in the Methods section. The optimized absorbance spectra for the results in Table 2 are shown in Fig. 4.

Fig. 4 shows the resulting absorbance spectra for point 1-4 after using the dielectric absorbance optimization procedure. In the optimized absorbers, the majority of the metallic absorbance has been transferred to the dielectric layer, and the maximum dielectric absorbance corresponds to the desired frequency. The information in Table 2 and Fig. 4 is useful for designing perfect absorbers for PV and other applications since a "map" of the absorber and material parameters can be constructed to guide the engineering of absorbers that achieve the maximum possible useful absorbance for any given semiconductor material used in place of the dielectric layer. The map resulting from the optimizations performed in this study can be seen in Fig. 5 .

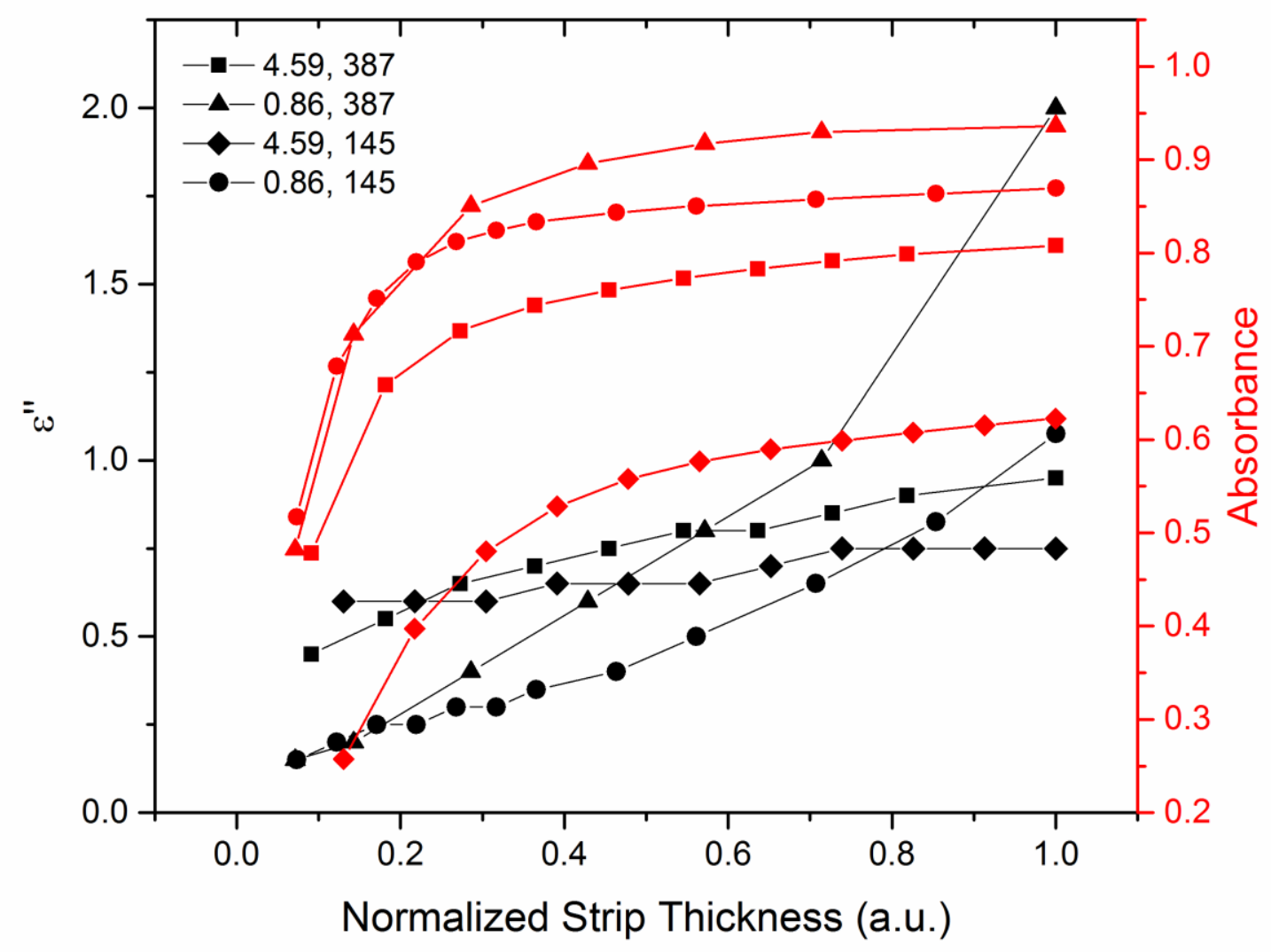

Figure 5. Plot of $\varepsilon^{\prime \prime}$ and absorbance versus normalized strip thickness. This plot can be used to guide the engineering of perfect absorbers for real semiconductor materials, with applications for PV and other solid state optical devices. Also, it can be seen that the absorbance converges or is very near convergence at the optimized $\varepsilon^{\prime \prime}$ for higher strip thicknesses, verifying that the optimization performed does in fact give approximately the highest achievable absorbance. This is explained by noting that the strip thickness is large enough for the skin depth to approach the bulk value and transfer nearly all of the metallic absorption to the dielectric layer. 
The plot in Fig. 5 displays the optimum imaginary component of the dielectric permittivity and the absorbance of the lossy dielectric layer as a function of strip thickness (normalized) for points 1-4 from Table 1. For a real permittivity of 4.59, as the resonant frequency goes from $145 \mathrm{THz}$ to $387 \mathrm{THz}$, it is observed that a larger imaginary permittivity is required to optimize the dielectric absorbance. For a real permittivity of 0.86 , the same trend is observed, but the difference between the imaginary components required for each frequency is larger than the higher real permittivity case. At the same time, the absorbance for the 0.86 case is higher than the 4.59 case over the whole frequency range, but a larger imaginary component of permittivity is required to optimize the absorbance at larger strip thicknesses. Also, it can be seen that the absorbance increases with increasing strip thickness. In other words, a thicker strip will drive more of the absorbance into the dielectric, which is expected as the skin depth approaches the bulk value. From this plot, it can be determined that a good candidate material for this type of absorbance enhancement would have relatively low real permittivity and high imaginary permittivity over the frequency range of $145-387 \mathrm{THz}(0.6-1.6 \mathrm{eV}$ or $2.07-0.77 \mu \mathrm{m})$, which corresponds to the near-IR regime. Also, the imaginary permittivity would preferably be larger at higher frequency. The approach presented here can be also applied to photodetectors and bulk metamaterials ${ }^{34,40-42}$ to minimize Ohmic losses.

\section{CONCLUSIONS}

The results of this study, which provided a map of $\varepsilon^{\prime \prime}$ and absorbance versus normalized strip thickness provide a guide for engineering of plasmonic perfect meta-absorbers for optical enhancement of PV and other optoelectronic applications. In order to apply optimized perfect absorbers to semiconductor materials commonly used in such devices, the permittivity as a function of frequency can be analyzed and compared to the map developed in here. While the near-IR regime studied here represents an appreciable amount of the solar spectrum, future work could include extension of the proposed perfect absorber optimization to the visible and UV regime, though it should be noted that this may be complicated by the appearance of higher order plasmonic modes. The presented approach can be also applied to photodetectors and bulk metamaterials to minimize Ohmic losses.

\section{ACKNOWLEDGMENT}

This work was supported by the National Science Foundation under grant Award No. CBET-1235750.

\section{REFERENCES}

[1] Gwamuri, J., Güney, D. Ö., and Pearce, J. M., Advances in Plasmonic Light Trapping in Thin-Film Solar Photovoltaic Devices, in Solar Cell Nanotechnology (eds A. Tiwari, R. Boukherroub and M. Sharon), John Wiley \& Sons, Inc., Hoboken, New Jersey, 241-269 (2013).

[2] Pendry, J. B., "Negative refraction makes a perfect lens," Phys. Rev. Lett. 85(18), 3966-3969 (2000).

[3] Xu, T., Zhao, Y., Ma, J., Wang, C., Cui, J., Du, C., and Luo, X., "Sub-diffraction-limited interference photolithography with metamaterials," Opt. Express 16(18), 13579-13584 (2008).

[4] Schurig, D., Mock, J. J., Justice, B. J., Cummer, S. A., Pendry, J. B., Starr, A. F., and Smith, D. R., "Metamaterial electromagnetic cloak at microwave frequencies," Science 314(5801), 977-980 (2006).

[5] Güney, D. O., and Meyer, D. A., "Negative refraction gives rise to the Klein paradox," Phys. Rev. A 79(6), 063834 (2009).

[6] Genov, D. A., Zhang, S., and Zhang, X., "Mimicking celestial mechanics in metamaterials," Nat. Phys. 5(9), 687-692 (2009).

[7] Bulu, I., Caglayan, H., Aydin, K., and Ozbay, E., "Compact size highly directive antennas based on the SRR metamaterial medium," New J. Phys. 7(1), 223 (2005).

[8] Odabasi, H., Teixeira, F., and Güney, D. O., "Electrically small, complementary electric-field-coupled resonator antennas," J. Appl. Phys. 113(8), 084903 (2013).

[9] Vora, A., Gwamuri, J., Pala, N., Kulkarni, A., Pearce, J. M., and Guney, D. O., "Exchanging Ohmic losses in metamaterial absorbers with useful optical absorption for photovoltaics," Sci. Rep. 4, 4901 (2014). 
[10] Vora, A., Gwamuri, J., Pearce, J. M., Bergstrom, P. L., and Guney, D. O., "Multi-resonant silver nano-disk patterned thin film hydrogenated amorphous silicon solar cells for Staebler-Wronski effect compensation," J. Appl. Phys. 116(9), 093103 (2014).

[11] Aslam, M. I. and Güney, D. O., “On negative index metamaterial spacers and their unusual optical properties,” Prog. Electromagn. Res. B 47, 203-217 (2013).

[12] Md. A. al Farooqui, J. Breeland, M. I. Aslam, M. Sadatgol, S. K. Ozdemir, M. Tame, L. Yang, and D. O. Guney, "Quantum entanglement distillation with metamaterials," Opt. Express 23(14), 17941 (2015).

[13] Béchu, M., Asano, M., Tame, M., Ozdemir, S. K., Ikuta, R., Yamamoto, T., Guney, D., Yang, L., Wegener, M., and Imoto, N., "Quantum entanglement distillation using an optical metamaterial," in CLEO: 2015, OSA Technical Digest (online) (Optical Society of America, 2015), paper FTu1A.8.

[14] Sihvola, A., "Metamaterials in electromagnetics," Metamaterials 1(1), 2-11 (2007).

[15] Pitarke, J. M., Silkin, V. M., Chulkov, E. V., and Echenique, P. M., “Theory of surface plasmons and surface-plasmon polaritons," Rep. Prog. Phys. 70(1), 1-87 (2007).

[16] Maier, S. A. and Atwater, H. A., "Plasmonics: localization and guiding of electromagnetic energy in metal/dielectric structures," J. Appl. Phys. 98(1), 011101 (2005).

[17] Maier, S. A., Plasmonics: Fundamentals and Applications, Springer, New York (2007).

[18] Halas, N. J., "Plasmonics: an emerging field fostered by Nano Letters," Nano Lett. 10(10), 3816-3822 (2010).

[19] Lal, S., Link, S., and Halas, N. J., "Nano-optics from sensing to waveguiding," Nat. Photon. 1(11), 641-648 (2007).

[20] Verellen, N., Sonnefraud, Y., Sobhani, H., Hao, F., Moshchalkov, V. V., Dorpe, P. V., Nordlander, P., and Maier, S. A., "Fano resonances in individual coherent plasmonic nanocavities," Nano Lett. 9(4), 1663-1667 (2009).

[21] Zayats, V., Smolyaninov, I. I., and Maradudin, A. A., "Nano-optics of surface plasmon polaritons," Phys. Rep. 408(3), 131-314 (2005).

[22] Guney, D. O., Koschny, Th., and Soukoulis, C. M., "Surface plasmon driven electric and magnetic resonators for metamaterials," Phys. Rev. B 83(4), 045107 (2011).

[23] Aslam, M. I. and Guney, D. O., "Surface plasmon driven scalable low-loss negative-index metamaterial in the visible spectrum,” Phys. Rev. B 84(19), 195465 (2011).

[24] Cui, Y., Xu, Y., Fung, K. H., Jin, Y., Kumar, A., He, S., and Fang, N. X., "A thin film broadband absorber based on multi-sized nanoantennas," Appl. Phys. Lett. 99(25), 253101 (2011).

[25] Wu, C. and Shvets, G., "Design of metamaterial surfaces with broadband absorbance," Opt. Lett. 37(3), 308-310 (2012).

[26] Hendrickson, J., Guo, J., Zhang, B., Buchwald, W., and Soref, R., "A wide-band perfect light absorber at mid-wave infrared using multiplexed metal structures," Opt. Lett. 37(3), 371-373 (2012).

[27] Alici, K. B., Turhan, A. B., Soukoulis, C. M., and Ozbay, E., "Optically thin composite resonant absorber at the near infrared band: polarization-independent and spectrally broadband configuration," Opt. Express 19(15), 14260-14267 (2011).

[28] Lin, C.-H., Chern, R.-L., and Lin, H.-Y., "Polarization-independent broad-band nearly perfect absorbers in the visible regime," Opt. Express 19(2), 415-424 (2011).

[29] Aydin, K., Ferry, V. E., Briggs, R. M., and Atwater, H. A., "Broadband polarization-independent resonant light absorption using ultrathin plasmonic super absorbers,” Nat. Commun. 2, 517 (2011).

[30] Jiang, Z.-H., Yun, S., Toor, F., Werner, D. H., and Mayer, T. S., “Conformal dual band near-perfectly absorbing midinfrared metamaterial coating," ACS Nano 5(6), 4641-4647 (2011).

[31] Diem, M., Koschny, Th., and Soukoulis, C. M., "Wide-angle perfect absorber/thermal emitter in the terahertz regime," Phys. Rev. B 79(3), 033101 (2009).

[32] Wang, J., Chen, Y., Hao, J., Yan, M., and Qiu, M., "Shape-dependent absorption characteristics of three-layered metamaterial absorbers at near-infrared,” J. Appl. Phys. 109(7), 074510 (2011).

[33] Guney, D. O., Koschny, T., and Soukoulis, C. M., "Reducing ohmic losses in metamaterials by geometric tailoring," Phys. Rev. B 80(12), 125129 (2009).

[34] Sadatgol, M., Ozdemir, S. K., Yang, L., and Guney, D. O., "Plasmon injection to compensate and control losses in negative index metamaterials," Phys. Rev. Lett. (in press).

[35] Hsu, L. and Walukiewicz, W. "Modeling of InGaN/Si tandem solar cells," J. Appl. Physics 104(2), 024507 (2008).

[36] Bhuiyan, A., Sugita, K., Hashimoto, A., and Yamamoto, A., "InGaN solar cells: present state of the art and important challenges," IEEE J. of Photovoltaics 2(3), 276-293 (2012).

[37] McLaughlin, D. V. P. and Pearce, J. M., "Progress in indium gallium nitride materials for solar photovoltaic energy conversion," Met. \& Mat. Trans. A 44(4), 1947-1954 (2013). 
[38] Hussain, S., Ali, G., Mehmood, H., Omar, M., and Zaidi, T., "Theoretical studies of InGaN/GaN multiple junction solar cell with enhanced tunneling junction diode,” Adv. Mat. Research 895, 535-538 (2014).

[39]Zhang, C., Gwamuri, J., Andrews, R., and Pearce. J. M., "Design of multijunction photovoltaic cells optimized for varied atmospheric conditions," Int. J. Photoenergy 2014, 514962 (2014).

[40] Guney, D. O., Koschny, Th., and Soukoulis, C. M., "Intra-connected three-dimensionally isotropic bulk negative index photonic metamaterial," Opt. Express 18(12), 12348-12353 (2010).

[41] Guney, D. O., Koschny, Th., Kafesaki, M. and Soukoulis, C. M., "Connected bulk negative index photonic metamaterials," Opt. Lett. 34(4), 506-508 (2009).

[42] Zhang, X., Debnath, S., and Guney, D. O., "Hyperbolic metamaterial feasible for fabrication with direct laser writing processes," J. Opt. Soc. Am. B 32(6), 1013 (2015). 